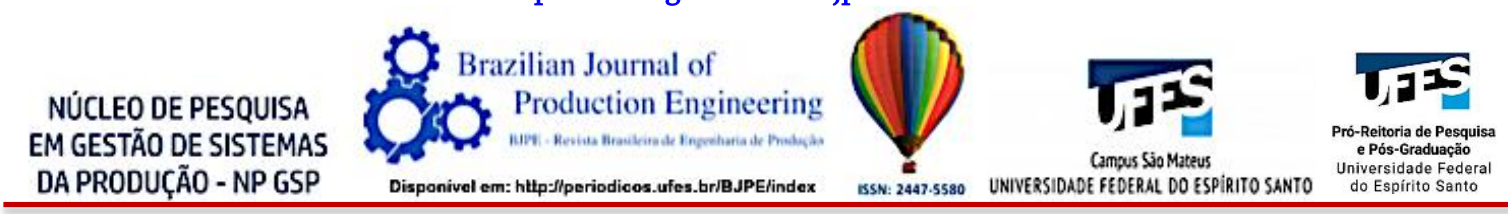

ARTIGO ORIGINAL

OPEN ACCESS

\title{
EFICÁCIA DO USO DOS CORTICOSTEROIDES COMO TERAPIA ADJUVANTE NO CHOQUE SÉPTICO
}

\section{EFFECTIVENESS OF THE USE OF CORTICOSTEROIDS AS ADJUVANT THERAPY IN SEPTIC SHOCK}

\author{
$\underline{\text { Wellington Felipe Jerônimo Leite }}{ }^{1 *}, \underline{\text { Osman Batista de Medeiros Filho }}{ }^{2}, \& \underline{\text { Milena }}$ \\ Nunes Alves de Sousa ${ }^{3}$ \\ ${ }^{123}$ Centro Universitário de Patos - UNIFIP. \\ 1* felippejeronimoleite@ gmail.com ${ }^{2}$ osmanbmf@ $\underline{\text { hotmail.com }}{ }^{3}$ milenanunes@ fiponline.edu.br
}

\section{ARTIGO INFO.}

\section{Recebido em: 29.12.2021}

Aprovado em: 21.02.2022

Disponibilizado em: 22.02.2022

\section{Palavras-chave:}

Eficácia; Corticosteroides; Terapêutica; Choque Séptico.

\section{KEYWORDS:}

Efficiency; Corticosteroids; Therapy; Septic shock.

*Autor Correspondente: Leite, W. F. J.

\section{RESUMO}

Contexto: $\mathrm{O}$ choque séptico consiste em um estado de falência circulatória aguda somada a um foco infeccioso ou com predomínio de componente endotóxico. Os corticosteroides são uma classe de fármacos sintetizados com base em hormônios esteroides endócrinos e tem sido usados com foco na hipoperfusão. Entretanto, a eficácia e a segurança da terapia com corticosteroides no choque séptico ainda permanecem incertas, visto que existem tanto relatos de melhora clínica quanto de efeitos adversos graves. Objetivo: Este estudo objetiva avaliar a eficácia da terapia adjuvante com corticosteroides em pacientes com choque séptico a fim de procurar se há evidências científicas que apresentem benefícios desse tratamento, bem como estabelecer, se positivo, o manejo, de acordo com as evidências, nessa patologia. Método: Caracteriza-se como uma Revisão Integrativa, a qual os artigos foram selecionados na plataforma da Biblioteca Virtual em Saúde, em que foram identificados 460 artigos, porém foram selecionados 39 dentre estes. Resultados: Esses estudos foram realizados com mais frequência nos anos de 2017, 2018 e 2019. Após a análise dos dados, sugeriu-se que essa terapêutica se relacionou com uma reversão mais rápida do choque, diminuição dos dias de uso de vasopressores e diminuem o tempo de internação em UTI. Contudo, existem riscos de efeitos adversos. Conclusão: Com isso, concluiu-se que os pacientes candidatos a esse tratamento são aqueles com maior gravidade e/ou que não respondem primeiramente a ressuscitação com fluidos e drogas vasoativas. Ademais o uso dessas medicações deve ser individualizado, levando-se em consideração o fenótipo e o genótipo de cada paciente.

\begin{abstract}
Context: Septic shock is a state of acute circulatory failure combined with an infectious focus or with a predominance of an endotoxic component. Corticosteroid therapy has been used with the main focus on hypoperfusion. Corticosteroids are a class of drugs synthesized on the basis of endocrine steroid hormones, which are produced and secreted by the cortex of the adrenal glands. However, the efficacy and safety of corticosteroid therapy in septic shock remains uncertain, as there are both reports of clinical improvement and serious adverse effects, such as superinfection and metabolic and neuromuscular effects. Objective: Given the above, the present study aims to evaluate the effectiveness of adjuvant therapy with corticosteroids in patients with septic shock in order to look for scientific evidence showing benefits of this treatment, as well as to establish, if positive, the management of according to the evidence, of corticosteroids in this pathology. Method: It is characterized as an Integrative Review, in which the articles were selected on the Virtual Health Library platform, in which 460 articles were identified, but after applying the filters and selection criteria, 39 were selected among them. Results: The country that was the target of studies on such behavior was the United States. These studies were carried out more frequently in 2017, 2018 and 2019. After analyzing the data, it was suggested that this therapy was related to a faster reversal of shock and a reduction in the days of vasopressor use, in addition to decreasing the length of stay in the ICU. However, there are risks of adverse effects. Conclusion: Therefore, it was concluded that patients who are candidates for this treatment are those with greater severity and/or who do not respond primarily to resuscitation with fluids and vasoactive drugs. Furthermore, the use of these medications must be individualized, considering the phenotype and genotype of each patient.
\end{abstract}




\section{INTRODUÇÃO}

O choque é uma condição clínica classificada como emergência médica que consiste em falência circulatória generalizada consequente ao dano à homeostase do oxigênio tissular, gerando desequilíbrio entre a oferta e a demanda desse gás (Jorge, Lourenço, Vieira, Santana, \& Pedroso, 2016). O estado clínico de choque consiste em uma falência circulatória e consequente baixa perfusão tecidual. Classicamente, o choque foi sistematizado pelos cientistas Blalock e Weil em cardiogênico, obstrutivo, hipovolêmico ou vasogênico, sendo o choque séptico a causa mais comum de choque não cardiogênico (Seymour \& Rosengart, 2015).

A sepse é definida como uma resposta orgânica e desregulada do hospedeiro frente a um processo infeccioso sistêmico, com risco de vida, que pode levar a sepse grave e ao choque séptico. Caracteriza-se pela presença de manifestações clínicas sistêmicas como hipotensão arterial, pulsos periféricos fracos, aumento do tempo de enchimento capilar, acidose láctica, oligúria, hipoxemia, frio distal e alterações neurológicas agudas (Garza et al., 2018). É responsável por cerca de 6 milhões de mortes neonatais e infantis por ano, respondendo por 60-80\% da mortalidade infantil anual, e seu estágio grave é o episódio intermediário entre a sepse e o choque séptico (Ismail \& Jayashree, 2018).

O choque séptico é classificado como um estágio avançado da sepse grave, que consiste em uma falha na resposta orgânica do hospedeiro ao estresse sistêmico, mediado por um processo inflamatório agressivo que se desenvolve no organismo (Meyer, et al., 2019) com taxa de mortalidade hospitalar superior a $40 \%$ e mais de 6 milhões de mortes anualmente, sendo considerada a principal causa de óbitos entre pacientes graves (Allen, Feild, Shoulders, \& Voils, 2019).

Nesta síndrome, os pacientes evoluem com hipotensão e hipoperfusão mesmo após tentativa de ressuscitação volêmica com fluidos, exigindo a administração de agentes vasopressores com o objetivo de estabilizar a pressão arterial média acima de $65 \mathrm{mmHg}$ e além disso, esses indivíduos apresentam uma concentração de lactato sérico maior do que $2 \mathrm{mmol} / \mathrm{L}$ (Cecconi, Evans, Levy, \& Rhodes, 2018).

De acordo com estudo etiopatológico, pode ser causado por diversos agentes microbiológicos, porém, os mais são comumente encontrados são as bactérias, como Staphylococcus aereus, Streptococcus pneumoniae ou Neisseria meningitidis. Entre os vírus, são frequentes o adenovírus e o citomegalovírus, seguidos pelos parasitas, destacando-se o Toxoplasma gondii. Por fim, os fungos, como a Candida sp (Garza et al., 2018).

O diagnóstico de sepse e choque séptico seguem critérios clínicos e laboratoriais que identificam disfunções orgânicas graves. Dentre estes temos rebaixamento do nível de consciência, agitação, delirium; hipotensão (PAS < $90 \mathrm{mmHg}$ ou PAM $<65 \mathrm{mmHg}$ ou queda da PA > $40 \mathrm{mmHg})$; oligúria $(\leq 0,5 \mathrm{ml} / \mathrm{Kg} / \mathrm{h})$ ou elevação da creatinina (> $2 \mathrm{mg} / \mathrm{dl})$; contagem de plaquetas $<100.000 / \mathrm{mm}^{3}$ ou redução de $50 \%$ da quantidade registrada nos últimos 3 dias; lactato acima do valor de referência; relação $\mathrm{PaO}_{2} / \mathrm{FiO}_{2}<300$ ou necessidade de $\mathrm{O}_{2}$ para manter a $\mathrm{SpO}_{2}>90 \%$; e aumento significativo de bilirrubinas (2 vezes maior que o valor de referência) (Instituto Latino Americano de Sepse, 2018). 
Citação (APA): Leite, W. F. J., Medeiros, O. B., de, Filho, \& Sousa, M. N. A., de. (2022). Eficácia do uso dos corticosteroides como terapia adjuvante no choque séptico. Brazilian Journal of Production Engineering, 8(1), 92-106.

É uma emergência hospitalar e o seu manejo adequado compreende o reconhecimento rápido e preciso dos sinais e sintomas clínicos, reversão imediata da hipoperfusão e a administração apropriada de antibióticos (Allen et al., 2019). Com foco principal na hipoperfusão, a corticoterapia adjuvante tem sido usada por mais de 40 anos, embora que atualmente a sua eficácia e segurança ainda permanecem incertas (Wen et al., 2019).

Os corticosteroides são uma classe de fármacos sintetizados com base em hormônios esteroides endócrinos, chamados de glicocorticoides, que são produzidos e secretados pelo córtex das glândulas adrenais. São assim nomeados em razão do seu mecanismo de ação no metabolismo dos carboidratos. Suas ações no metabolismo da glicose podem elevar os níveis da glicemia de jejum a partir do aumento da resistência à insulina (Ramamoorthy \& Cidlowski, 2016).

Além da ação no metabolismo da glicose, também apresentam ação anti-inflamatória e imunossupressora quando são administrados em altas doses farmacológicas. A primeira ocorre a partir do estimulo para a produção da lipocortina, uma inibidora da enzima fosfolipase $\mathrm{A}_{2}$, diminuindo a produção do ácido araquidônico, que é um precursor dos mediadores inflamatórios locais, sendo estes os leucotrienos, prostaglandinas e tromboxanos, que realizam a dilatação dos capilares, aumentam a permeabilidade vascular e estimulam a migração de leucócitos para as áreas de lesão tissular. Já a supressão do sistema imune se dá por meio da diminuição da produção de linfócitos T e anticorpos, fato que decorre da inibição da síntese de interleucina-2 (IL-2), um metabolito essencial para a imunidade celular (Hall \& Guyton, 2017). Por conseguinte, uma reação potencialmente grave é o aumento da suscetibilidade à infecção e às lesões (Golan, Tashjian, Armstrong, \& Armstrong, 2018).

Atuam, ainda, na manutenção da resposta vascular às catecolaminas, tendo um papel permissivo para estas nas arteríolas por meio da estimulação dos receptores $\alpha 1$-adrenérgicos. Deste modo, o cortisol é importante para a regulação da pressão arterial sistêmica, visto que facilita a atividade vasoconstritora das catecolaminas nas arteríolas. Além disso, estes esteroides apresentam efeitos sob o sistema nervoso central (SNC), diminuindo o sono REM e aumentando o tempo de vigília, e também no sistema esquelético, realizando a inibição da osteogênese a partir da diminuição da síntese de colágeno tipo I, principal componente da matriz óssea; redução da absorção intestinal de cálcio $\left(\mathrm{Ca}^{2+}\right)$; e minimizando a formação de osso novo pelos osteoblastos (Costanzo, 2018).

Devido à suas ações sistêmicas, os corticosteroides são responsáveis por vários efeitos adversos graves que dependem fundamentalmente da duração do tratamento e da dose farmacológica consumida. Considerando-se que os glicocorticoides endógenos influenciam em processos imunes, estes efeitos intensificam-se após o uso de doses farmacológicas dessas drogas, causando os efeitos adversos (Ritter et al., 2020).

Ante as ponderações, como questão norteadora indaga-se qual é a eficácia do uso dos corticosteroides como terapia adjuvante no choque séptico? Uma vez que catalogá-la permite um desfecho mais favorável.

A eficácia e a segurança da terapia com corticosteroides no choque séptico ainda permanecem incertas. Portanto, este estudo visa avaliar a eficácia da terapia adjuvante com corticosteroides 

adjuvante no choque séptico. Brazilian Journal of Production Engineering, 8(1), 92-106.

em pacientes com choque séptico a fim de procurar se há evidências científicas que apresentem benefícios desse tratamento, bem como estabelecer, se positivo, o manejo, de acordo com as evidências, dos corticosteroides nessa patologia.

\section{MATERIAL E MÉTODOS}

Com a finalidade de atender ao objetivo deste estudo, adotou-se como método a revisão integrativa da literatura. Trata-se de uma metodologia que viabiliza a condensação do conhecimento e a integração da aplicabilidade de resultados de estudos significativos na prática. Este método faz parte da Prática Baseada em Evidência (PBE), descrita pelo epidemiologista Archie Cochrane, visto que envolve o estabelecimento de um problema clínico, o reconhecimento das informações mais essenciais, a realização de uma pesquisa de estudos na literatura e sua consequente avaliação crítica, a exposição da aplicabilidade dos dados provenientes das publicações e a determinação de sua utilização para o paciente (Souza, Silva, \& Carvalho, 2010).

Sua efetivação consiste no ato de percorrer por seis fases. Segundo Mendes, Silveira e Galvão (2010), a primeira etapa abrange tanto a elaboração de um tema, quanto de uma questão norteadora da pesquisa. Na segunda realiza-se a determinação dos fatores de inclusão e exclusão dos estudos. Sucessivamente, deve ser feita a síntese das informações a serem retiradas dos estudos selecionados. Na quarta etapa, faz-se a análise dos estudos inseridos na revisão integrativa. Posteriormente, na quinta etapa, desenvolvem-se os resultados. Finalmente, faz-se a exposição da revisão/síntese do conhecimento.

Seguindo-se as fases descritas, incialmente foi feita a elaboração do tema de estudo "Eficácia do uso dos corticosteroides como terapia adjuvante no choque séptico", juntamente com a questão de norteadora da pesquisa "Qual é a eficácia do uso dos corticosteroides como terapia adjuvante no choque séptico?".

Posteriormente, na fase dois, a base de dados para seleção dos estudos foi escolhida e os critérios de inclusão e exclusão foram estabelecidos. A coleta dos dados, realizada nos meses de março, abril e maio de 2021, foi executada na plataforma do Portal Regional da Biblioteca Virtual em Saúde (BVS), https://bvsalud.org, utilizando-se das palavras de pesquisa "Corticosteroides" "Choque Séptico". A partir disso, foram encontrados 425 artigos na base de dados da Medical Literature Analysis and Retrieval System Online (MEDLINE), 19 artigos na Literatura Latino-Americano e do Caribe em Ciências da Saúde (LILACS), 13 artigos no Índice Bibliográfico Espanhol das Ciências da Saúde (IBECS) e 2 artigos na Base Internacional de Guias GRADE (BIGG), totalizando 460 publicações.

Por conseguinte, ainda nessa etapa, foram estabelecidos os critérios de inclusão, que consistem em espaço temporal de 2016 a 2021, publicações com assunto principal "Corticosteroides" "Choque Séptico" e publicados em inglês e espanhol, visto que nenhum foi encontrado na língua portuguesa. Ademais, também se utilizou de critérios de exclusão, quando foram excluídos os artigos repetidos, considerando-os apenas uma vez, e os que foram considerados que não respondiam à questão norteadora do estudo. Dessa forma, identificaram-se os estudos pré-selecionados e selecionados (Figura 1).

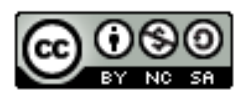



adjuvante no choque séptico. Brazilian Journal of Production Engineering, 8(1), 92-106.

Figura 1. Fluxograma relacionado ao processo de seleção dos estudos.

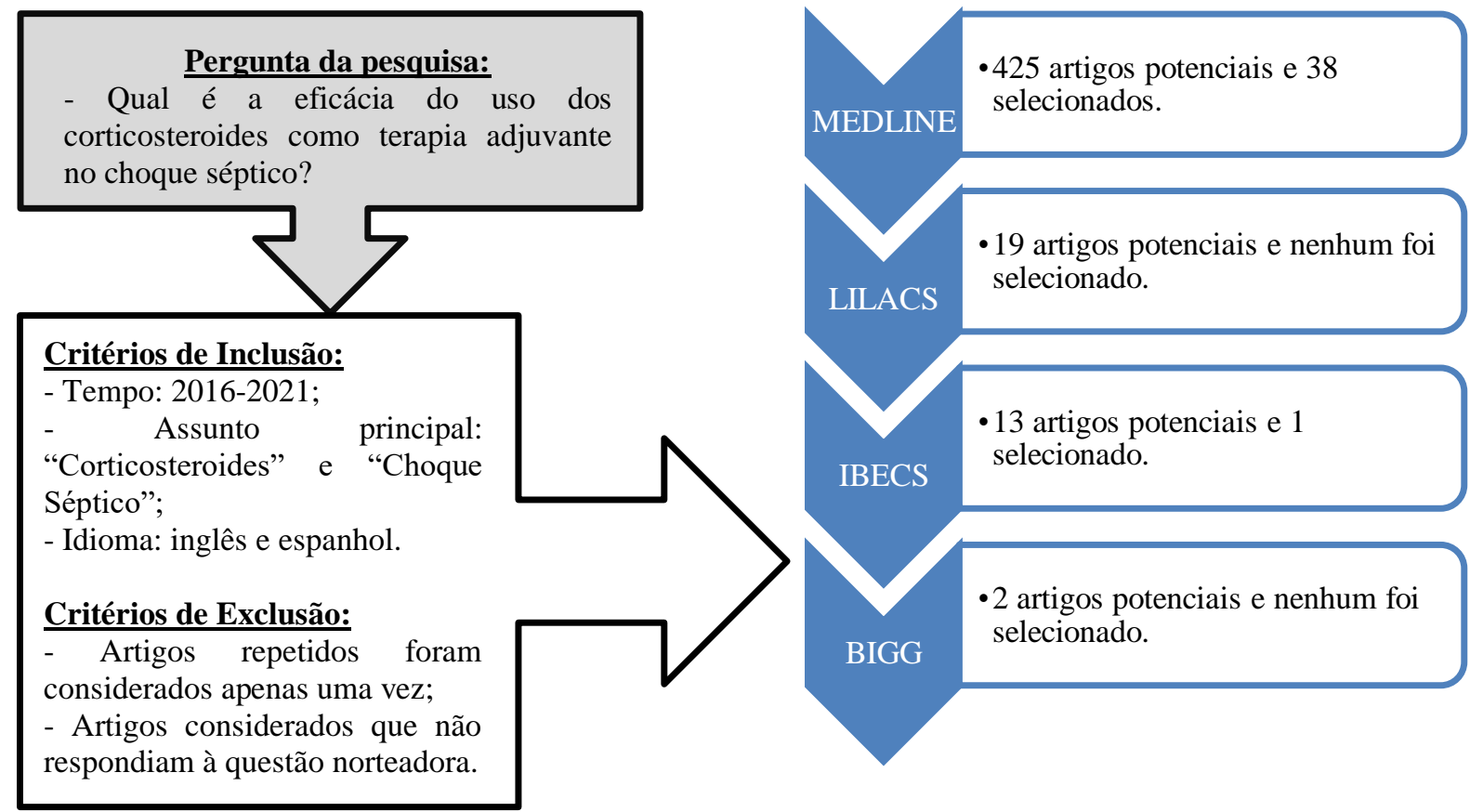

Os dados provenientes do passo a passo para a coleta de dados a serem utilizados serão categorizados em um fluxograma e em um quadro de análise, em que os estudos selecionados foram comparados por meio de categorias como: principais ações da classe farmacológica estudada, seus efeitos adversos, estudos a favor e contra seu uso no choque séptico, país onde o estudo foi realizado, entre outros. A análise dos dados, por sua vez, será efetivada por meio de uma visão qualitativa.

\section{RESULTADOS}

De acordo com o ano de publicação, contatou-se um aumento significativo do número de produções de artigos novos, considerando o número de 39 escritos que foram selecionados, ao longo dos anos, evidenciando a atualidade do tema e a necessidade de ser melhor entendido. Dentre esse período, o ano de 2018 contabilizou a maior parte dos trabalhos selecionados, totalizando 12 artigos publicados (30,7\%) (Gráfico 1).

Gráfico 1. Anos de publicação dos artigos
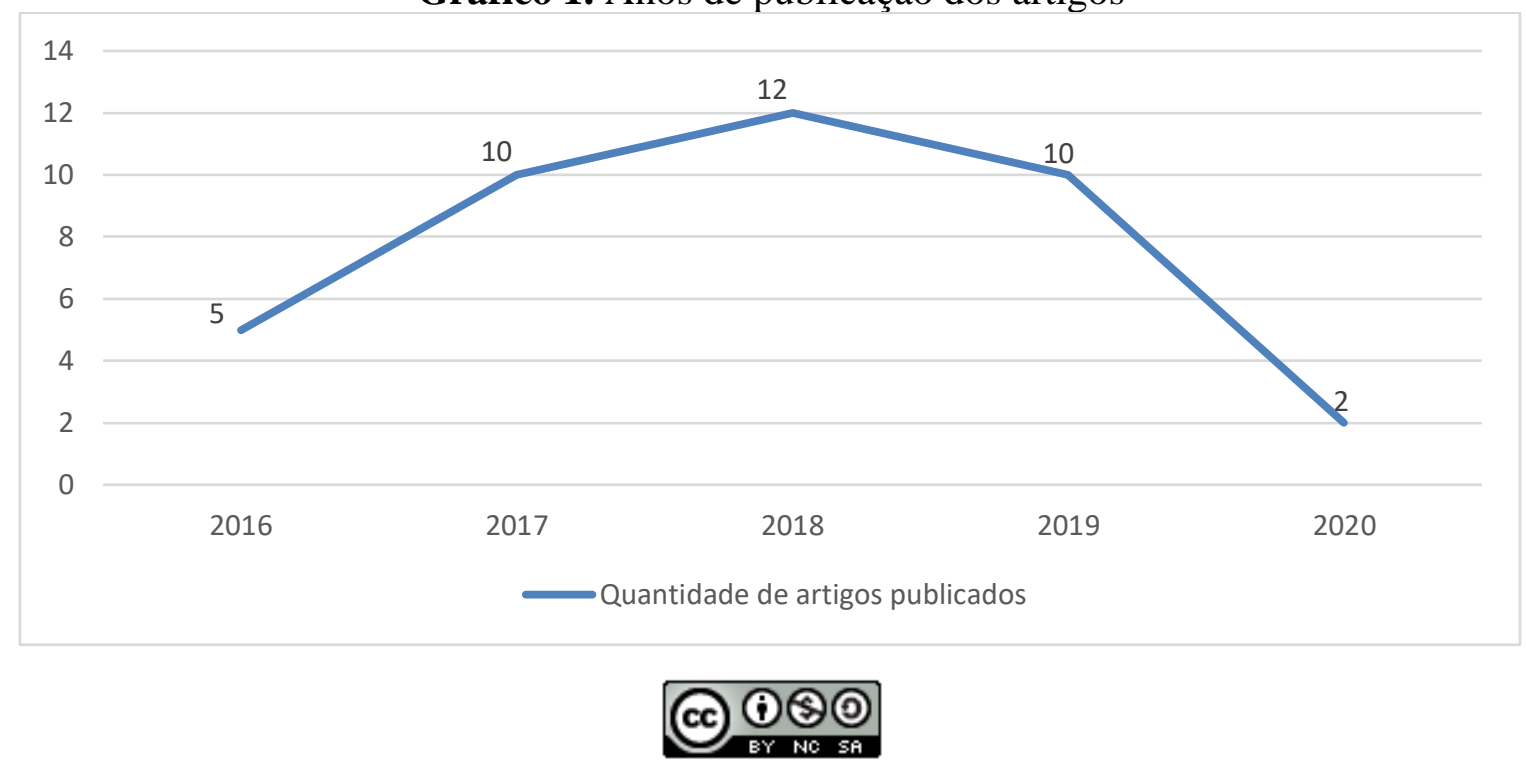
Citação (APA): Leite, W. F. J., Medeiros, O. B., de, Filho, \& Sousa, M. N. A., de. (2022). Eficácia do uso dos corticosteroides como terapia adjuvante no choque séptico. Brazilian Journal of Production Engineering, 8(1), 92-106.

Quanto ao país de origem da publicação, registrou-se a realização dos 39 artigos distribuídos em 13 países, com predomínio significante dos Estados Unidos, atingindo 11 trabalhos $(28,2 \%)$, e em segundo encontram-se Canadá e China, ambos com 6 publicações $(15,3 \%)$. Devido a essa postura, estes representam os países com melhores qualidades e desenvolvimento dos cuidados em saúde. Por outro lado, encontra-se, por exemplo, o Brasil, representado apenas por 1 artigo (2,5\%), mostrando a necessidade que existe acerca de mais produções cientificas sobre o tema. Considerando o tipo e o cenário de estudo, o estudo quantitativo e a área hospitalar foram totalmente dominante (100\%), haja vista que o tema proposta é obrigatoriamente uma causa de internação hospitalar para cuidado imediato e por ser necessário a busca de números para que seja feita uma conduta adequada com protocolos (Quadro 1).

Quadro 1. Caracterização referente ao título, país, cenário do estudo e tipo do estudo.

\begin{tabular}{|c|c|c|c|}
\hline Titulo & País & $\begin{array}{l}\text { Cenário } \\
\text { de Estudo }\end{array}$ & $\begin{array}{l}\text { Tipo de } \\
\text { Estudo }\end{array}$ \\
\hline $\begin{array}{l}\text { A depleção total e de alta afinidade da globulina de ligação ao corticosteroide } \\
\text { no choque séptico está associada à mortalidade. }\end{array}$ & Austrália & Hospitalar & Quantitativo \\
\hline $\begin{array}{l}\text { A eficácia e segurança da terapia com corticosteroides em pacientes críticos } \\
\text { adultos com choque séptico: uma meta-análise de ensaios clínicos } \\
\text { randomizados. }\end{array}$ & China & Hospitalar & Quantitativo \\
\hline $\begin{array}{l}\text { A resposta inadequada ao teste de estimulação com cosintropina é uma } \\
\text { indicação de resistência aos corticosteroides no choque séptico? }\end{array}$ & França & Hospitalar & Quantitativo \\
\hline $\begin{array}{l}\text { Administração de corticoides nos pacientes com sepse grave e mejor de sua } \\
\text { mortalidade intrahospitalaria: uma revisão sistemática. }\end{array}$ & Espanha & Hospitalar & Quantitativo \\
\hline $\begin{array}{l}\text { Associação do tratamento com corticosteroides com desfechos em pacientes } \\
\text { adultos com sepse. }\end{array}$ & China & Hospitalar & Quantitativo \\
\hline Atualização em corticosteróides de baixa dosagem. & Alemanha & Hospitalar & Quantitativo \\
\hline $\begin{array}{l}\text { Atualizações recentes no manejo farmacológico da sepse e choque séptico: } \\
\text { uma revisão sistemática focada em ressuscitação volêmica, vasopressores e } \\
\text { corticosteroides. }\end{array}$ & EUA & Hospitalar & Quantitativo \\
\hline $\begin{array}{l}\text { Avaliação da corticoterapia precoce no manejo do choque séptico pediátrico } \\
\text { em pacientes pediátricos de terapia intensiva: um estudo clínico randomizado. }\end{array}$ & Egito & Hospitalar & Quantitativo \\
\hline $\begin{array}{l}\text { Avaliando a viabilidade de ensaios clínicos randomizados de corticosteroides } \\
\text { em choque séptico pediátrico em países desenvolvidos: apenas metade da } \\
\text { resposta ao problema para o editor. }\end{array}$ & Índia & Hospitalar & Quantitativo \\
\hline $\begin{array}{c}\text { Avanços no manejo do choque séptico pediátrico: velhas questões, novas } \\
\text { respostas. }\end{array}$ & Índia & Hospitalar & Quantitativo \\
\hline $\begin{array}{l}\text { Combinando estratégias de enriquecimento prognóstico e preditivo para } \\
\text { identificar crianças com choque séptico responsivas a corticosteroids. }\end{array}$ & EUA & Hospitalar & Quantitativo \\
\hline $\begin{array}{l}\text { Contribuições de uma pesquisa e estudo de coorte retrospectivo para o } \\
\text { planejamento de um estudo controlado randomizado de corticosteroides no } \\
\text { tratamento do choque séptico pediátrico. }\end{array}$ & Canadá & Hospitalar & Quantitativo \\
\hline $\begin{array}{l}\text { Controvérsias na aplicação de corticosteroides para tratamento de choque } \\
\text { séptico pediátrico: itens de notificação preferidos para revisões sistemáticas e } \\
\text { metanálise atualizada compatível com meta-análise. }\end{array}$ & China & Hospitalar & Quantitativo \\
\hline Controvérsias no uso de corticosteroides para sepse. & EUA & Hospitalar & Quantitativo \\
\hline $\begin{array}{l}\text { Corticosteroides adjuvantes para choque pediátrico contemporâneo: análise } \\
\text { contínua da relação risco-benefício. }\end{array}$ & EUA & Hospitalar & Quantitativo \\
\hline $\begin{array}{l}\text { Corticosteroides em baixas doses e resolução de choque séptico: implicações } \\
\text { para o desenho de estudos randomizados? }\end{array}$ & EUA & Hospitalar & Quantitativo \\
\hline Corticosteroides na sepse grave e choque séptico: uma revisão concise. & Portugal & Hospitalar & Quantitativo \\
\hline Corticosteroides no choque séptico pediátrico não são úteis. & EUA & Hospitalar & Quantitativo \\
\hline Corticosteroides no choque séptico pediátrico são úteis. & Canadá & Hospitalar & Quantitativo \\
\hline Corticosteroides no choque séptico: qual deve ser a decisão em pediatria? & Brasil & Hospitalar & Quantitativo \\
\hline $\begin{array}{l}\text { Corticosteroides no choque séptico: uma revisão sistemática e metanálise em } \\
\text { rede. }\end{array}$ & Inglaterra & Hospitalar & Quantitativo \\
\hline Corticoterapia adjuvante no choque séptico. & Austrália & Hospitalar & Quantitativo \\
\hline $\begin{array}{l}\text { Efeito do ácido ascórbico, corticosteroides e tiamina na lesão de órgãos no } \\
\text { choque séptico: ensaio clínico randomizado de atos. }\end{array}$ & EUA & Hospitalar & Quantitativo \\
\hline
\end{tabular}


Citação (APA): Leite, W. F. J., Medeiros, O. B., de, Filho, \& Sousa, M. N. A., de. (2022). Eficácia do uso dos corticosteroides como terapia adjuvante no choque séptico. Brazilian Journal of Production Engineering, 8(1), 92-106.

\begin{tabular}{|c|c|c|c|}
\hline $\begin{array}{l}\text { Ensaios clínicos randomizados de corticosteróides no choque séptico: } \\
\text { possivelmente viável, mas eles vão ou devem mudar minha prática? }\end{array}$ & Canadá & Hospitalar & Quantitativo \\
\hline Esteroides e sepse: o debate continua. & Austrália & Hospitalar & Quantitativo \\
\hline $\begin{array}{l}\text { Esteróides em choque pediátrico dependente de infusão de fluidos e/ou } \\
\text { vasoativos: protocolo de estudo para um ensaio clínico randomizado. }\end{array}$ & Canadá & Hospitalar & Quantitativo \\
\hline $\begin{array}{c}\text { Níveis plasmáticos de citocinas predizem resposta a corticosteroides no } \\
\text { choque séptico. }\end{array}$ & Suécia & Hospitalar & Quantitativo \\
\hline O papel controverso dos corticosteroides no choque séptico. & EUA & Hospitalar & Quantitativo \\
\hline $\begin{array}{l}\text { O papel do corticoide em pacientes com choque séptico: os autores } \\
\text { respondem. }\end{array}$ & China & Hospitalar & Quantitativo \\
\hline O papel do corticosteróide em pacientes com choque séptico. & China & Hospitalar & Quantitativo \\
\hline $\begin{array}{c}\text { O tratamento adjuvante com corticosteroides em pacientes críticos com } \\
\text { choque séptico (adrenal) foi insuficiente? }\end{array}$ & EUA & Hospitalar & Quantitativo \\
\hline $\begin{array}{l}\text { Os corticosteroides melhoram a mortalidade ou a reversão do choque em } \\
\text { pacientes com choque séptico? }\end{array}$ & EUA & Hospitalar & Quantitativo \\
\hline $\begin{array}{c}\text { Para uma maior compreensão da controvérsia dos esteróides no choque } \\
\text { séptico. }\end{array}$ & Canadá & Hospitalar & Quantitativo \\
\hline $\begin{array}{c}\text { Polimorfismos do receptor de glicocorticóides e desfechos no choque séptico } \\
\text { pediátrico. }\end{array}$ & EUA & Hospitalar & Quantitativo \\
\hline $\begin{array}{c}\text { Por que entender os endótipos de sepse é importante para estudos de } \\
\text { esteroides no choque séptico. }\end{array}$ & Inglaterra & Hospitalar & Quantitativo \\
\hline Por que meus testes de esteróides em choque séptico foram "positivos". & França & Hospitalar & Quantitativo \\
\hline $\begin{array}{l}\text { Por que o tratamento adjuvante com corticosteroides em pacientes críticos } \\
\text { com choque séptico (adrenal) não mostrou diferença na mortalidade. }\end{array}$ & Austrália & Hospitalar & Quantitativo \\
\hline $\begin{array}{l}\text { Quando e como usar biomarcadores preditivos para o tratamento do choque } \\
\text { séptico com corticosteroides. }\end{array}$ & Canadá & Hospitalar & Quantitativo \\
\hline $\begin{array}{c}\text { Reavaliando o papel dos corticosteroides no choque séptico: uma metanálise } \\
\text { atualizada de ensaios clínicos randomizados. }\end{array}$ & China & Hospitalar & Quantitativo \\
\hline
\end{tabular}

A Figura 2 sintetiza uma tríade guiada pelas principais temáticas que guiaram os estudos selecionados.

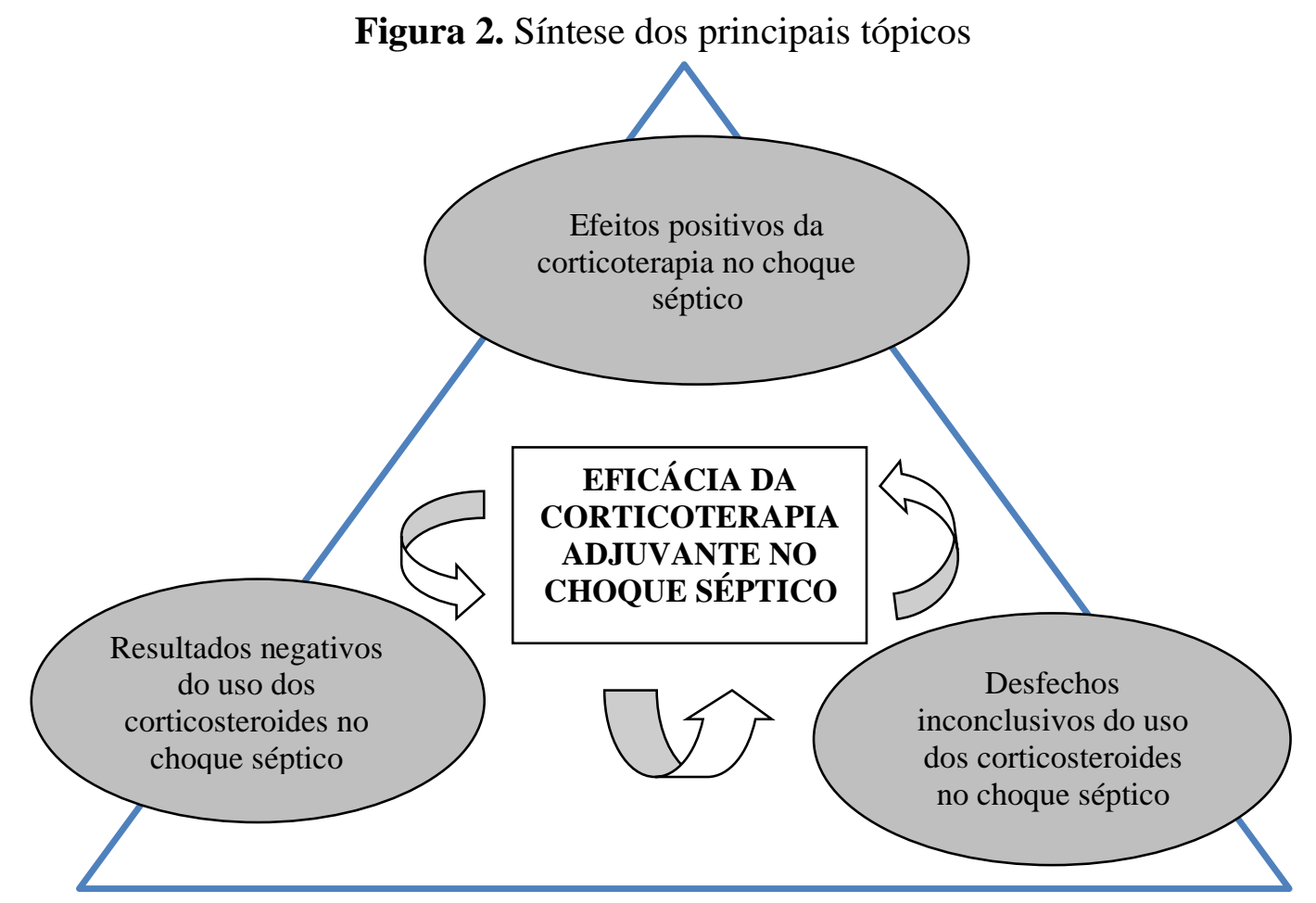

\section{DISCUSSÃO}

O manejo terapêutico do choque séptico é influenciado por vários fatores que contribuem para diminuição da taxa de letalidade, incluindo diagnóstico precoce e intervenção imediata. O protocolo de tratamento dessa síndrome geralmente desenvolve-se por meio de resgate, 
Citação (APA): Leite, W. F. J., Medeiros, O. B., de, Filho, \& Sousa, M. N. A., de. (2022). Eficácia do uso dos corticosteroides como terapia adjuvante no choque séptico. Brazilian Journal of Production Engineering, 8(1), 92-106.

otimização e estabilização. Por conseguinte, geralmente os pacientes recebem acesso venoso imediato para administração de fluidos, vasopressores e cuidados visando recompor a circulação normal (Seymour \& Rosengart, 2015).

A utilização dos corticosteroides na terapia do choque séptico ainda permanece incerta nos dias atuais, entretanto, sabe-se que essas drogas são recomendadas para pacientes com choque séptico refratário, ou seja, aqueles em que a pressão arterial alvo não é atingida e/ou não se mantem estável, mesmo após ressuscitação volêmica e uso de vasopressores. Contudo, mesmo sem este critério, quando se considera o tempo de ventilação mecânica e o tempo de internação em UTI, alguns pacientes se beneficiam dessas drogas, por isso, seu uso deve ser individualizado. A droga mais aconselhada é a hidrocortisona na dose de $5 \mathrm{mg}$ a cada 6 horas (Instituto Latino Americano de Sepse, 2018).

Conforme o estudo realizado, os resultados encontrados foram divididos em: artigos que mostraram efeitos e resultados positivos da corticoterapia adjuvante no choque séptico (Annane, 2019; Antcliffe \& Gordon, 2019; Cohen \& Venkatesh, 2019; El-Nawawy, Khater, Omar, \& Wali, 2017; Fang et al., 2018; Garza et al., 2018; Gibbison et al., 2017; Law, Sahetya, Walkey, \& Delaney, 2018; Lee \& Yeh, 2018; Lian et al., 2019; Liu \& Menon, 2018; Menon \& Mcnally, 2018; Meyer et al., 2019; Pourmand, Whiteside, Yamane, Rashed, \& Mazer-Amirshahi, 2019; Russell, 2018. Wen et al., 2019; Wong et al., 2016; Zhou, Ye, Li, \& Tang, 2018), e resultados negativos do uso complementar dos corticosteroides no choque séptico (Cvijanovich et al., 2017; Lemieux, Levine, \& Gritsenko, 2019; Long \& Koyfman, 2017; Moskowitz et al., 2020; Salluh \& Póvoa, 2017; Venkatesh \& Cohen, 2019; Yang \& Sun, 2020; Zimmerman, 2018).

Também foram apresentados estudos com desfechos inconclusivos do uso auxiliar dos corticosteroides no choque séptico (Allen et al., 2019; Angurana \& Kumar, 2017; Argent \& Kissoon, 2017; Bentzer, Fjell, Walley, Boyd, \& Russell, 2016; Briegel, Bein, \& Mohnle, 2017; Darmaros, Delgado, \& Carvalho, 2016; Hyvernat et al., 2017; Ismail \& Jayashree, 2018; Long \& April, 2018; O’Hearn et al., 2016; Ramanan, Cohen, \& Venkatesh, 2019; Russell \& Sevransky, 2019; Zimmerman, 2016).

Segundo as diretrizes atuais da Surviving Sepsis Campaign, a corticoterapia no choque séptico deve ser instituída em pacientes que não tiveram uma restauração adequada da estabilidade hemodinâmica após o uso de fluidos e tratamento com vasopressores. Após a análise dos dados renovados de ensaios clínicos randomizados (ECRs), sugeriu-se que o tratamento com corticosteroides se associou à redução da mortalidade em comparação com o controle em pacientes sépticos. Ademais, essa terapêutica relacionou-se com uma maior reversão do choque e diminuição dos dias de uso de vasopressores, além de diminuir o tempo de internação em UTI. Com isso, esses achados sugerem que os corticoides podem ser prescritos em doses baixas e em longo curso. No entanto, estudos futuros são necessários para que se tenha um controle adequado acerca dos pacientes que são candidatos ou não a receber este tratamento adjuvante (Fang et al., 2019).

A fundamentação de suporte para efeitos potencialmente benéficos da corticoterapia adjuvante no choque séptico inclui estudos em animais e humanos, raciocínio fisiológico, 
Citação (APA): Leite, W. F. J., Medeiros, O. B., de, Filho, \& Sousa, M. N. A., de. (2022). Eficácia do uso dos corticosteroides como terapia adjuvante no choque séptico. Brazilian Journal of Production Engineering, 8(1), 92-106.

recomendações de diretrizes e comparação de seu uso em outros cuidados intensivos. O cortisol é um hormônio pleotrópico que contribui significativamente para a manutenção da estabilidade hemodinâmica. Ele apresenta ações não genômicas imediatas, por meio da redução da receptação de norepinefrina, fazendo com que os receptores $\beta$-adrenérgicos no coração fiquem mais sensíveis e aumentando a disponibilidade de cálcio nas células do músculo liso do miocárdio e vascular, intensificando a contratilidade miocárdica e gerando vasoconstrição (Menon \& Mcnally, 2018).

Além disso, esses medicamentos também exercem efeitos genômicos, inibindo a produção da enzima óxido nítrico sintetase e da prostaciclina, causando um aumento do tônus vascular, estimulando fatores de adesão intercelulares do músculo liso vascular e aumentando o número de receptores $\beta$-adrenérgicos no coração, resultando em aumento do cronotropismo cardíaco. A deficiência deste hormônio em nível celular contribui para a instabilidade hemodinâmica devido à redução da contratilidade miocárdica, aumento da vasodilatação e/ou síndrome de vazamento capilar (Menon \& Mcnally, 2018).

Nos últimos anos ocorreram vários debates sobre qual é o momento ideal para iniciar a corticoterapia e como é a melhor forma de administrá-la no choque séptico, seja precoce ou tardiamente, em bomba de infusão contínua ou em bolus. Após estudos de revisão, concluiuse que a corticoterapia adjuvante influencia no benefício do tratamento, visto que o tempo de início da terapia é menor, nas seis primeiras horas após hipotensão, entre os sobreviventes em comparação àqueles que morreram. No entanto, este estudo apresentou limitações por ser retrospectivo, possibilitando a ocorrência de viés de seleção. Em relação a forma de administração do corticoide, notou-se uma restauração mais precoce do choque e desmame mais precoce das drogas vasoativas nos pacientes que fizeram uso de bomba de infusão em comparação ao bolus (Garza et al., 2018).

Um fator importante do choque séptico que pode estimular a administração de corticosteroides é a velocidade com que esse quadro pode progredir para morte. A partir de um estudo observacional recente nos Estados Unidos, evidenciou-se que cerca de $25 \%$ das mortes por choque séptico ocorrem nas primeiras 24 horas após o diagnóstico, demonstrando que muitas vezes apenas a terapia padrão (fluidos, antibióticos e inotrópicos) não é eficaz. Ademais, o American College of Critical Care Medicine recomenda que caso um paciente apresente risco de insuficiência adrenal absoluta ou falência no eixo hipófise-adrenal e após a administração de catecolaminas permanece em choque, este deve receber infusão de corticoide. (Menon \& Mcnally, 2018).

Por outro lado, após o estudo de algumas metanálises notou-se que a corticoterapia adjuvante no choque séptico em alguns casos pode associar-se a vários efeitos adversos, incluindo fraqueza neuromuscular, desordens metabólicas, imunossupressão e sangramento gastrointestinal. No entanto, a adversidade encontrada mais frequentemente foi a hiperglicemia e hipernatremia, seguida de um pequeno aumento na taxa de fraqueza neuromuscular. Além disso, as estatísticas de ocorrência de sangramento gastrointestinal ou superinfecção não tiveram aumento. Com isso, notou-se que a administração de 
Citação (APA): Leite, W. F. J., Medeiros, O. B., de, Filho, \& Sousa, M. N. A., de. (2022). Eficácia do uso dos corticosteroides como terapia adjuvante no choque séptico. Brazilian Journal of Production Engineering, 8(1), 92-106.

corticosteroides se associa a um aumento dos distúrbios metabólicos menores, porém a ocorrência de efeitos adversos graves é baixa (Cohen \& Venkatesh, 2019).

Segundo estudos recentes, o papel da corticoterapia em pacientes com choque séptico resistente ao tratamento com vasopressores permanece controverso e muito incerto, principalmente no que tange à redução de mortalidade, superinfecção e redução do tempo de choque. Diante disto, não há orientação clara sobre quando os corticosteroides devem ser administrados, bem como qual tipo de paciente se beneficiaria (Long \& Koyfman, 2017).

A corticoterapia nos pacientes com choque séptico geralmente é realizada nos quadros que se encontram em estado mais crítico, quando o efeito corticoide permissivo às catecolaminas nos vasos pode auxiliar na manutenção da pressão arterial sistêmica. Além disso, como atualmente os corticosteroides são considerados também imunomoduladores, essas drogas podem ajudar a controlar a resposta inflamatória sistêmica exacerbada que ocorre durante o choque séptico, reduzindo possivelmente a gravidade do quadro. Entretanto, essas drogas aumentam o risco de infecções oportunistas, principalmente em altas doses, visto que os pacientes já se encontram debilitados (Garza et al., 2018).

Um dos maiores efeitos negativos dos corticosteroides é que estes afetam vários órgãos com estreita regulação intrínseca. Com isso, quantidades em excesso dessas substancias podem causar eventos adversos que variam de leve a muito graves, como hiperglicemia, hipernatremia, sangramento gastrointestinal, infecção secundária por imunossupressão, cura retardada, ruptura da pele e fraqueza muscular devido à miopatia. A ocorrência de glicemia sérica elevada é a mais comum e geralmente não causa danos graves aos pacientes, visto que é controlada com o uso de insulina, contudo, esse medicamento pode causar dano pelo maior risco de hipoglicemia. Com relação aos pacientes internados na unidade de terapia intensiva (UTI), o risco de adversidades mais graves é sempre maior como, por exemplo, a mioneuropatia de doença crítica, fraqueza prolongada, maior tempo de internação na UTI e ventilação mecânica prolongada (Long \& Koyfman, 2017).

Um estudo com 18 ensaios clínicos randomizados, incluindo 8.128 pacientes em estado de choque séptico crítico, mostrou que a corticoterapia adjuvante não teve ação redutora significativa na mortalidade durante 28 dias. Além disso, levando em consideração os 90 dias, a mortalidade na UTI e a intra-hospitalar, o tempo de permanência hospitalar, a reversão do choque e a superinfecção não tiveram diferença significativa entre a terapia com corticosteroides e a terapia com placebo. Esses resultados foram obtidos ao considerar dosagens tanto baixas quanto altas de corticoides (Wen et al., 2019).

Um dos principais problemas sobre o uso dos corticosteroides no choque séptico é a resposta diferente que cada paciente apresenta após a administração, visto que dependem dos perfis genômicos, transcriptômicos e proteômicos individuais. Recentemente, levantarem-se hipóteses de que um receptor, chamado receptor leucocorticoide periférico (GCR) e os níveis de cortisol sérico se relacionam com a resposta à corticoterapia. Nesse estudo, os biomarcadores foram medidos e os níveis de expressão de GCR foram menores e os níveis de cortisol sérico foram maiores em pacientes que tiveram resultados piores (Russell, 2018).

\section{@ $\odot \odot \odot$}



adjuvante no choque séptico. Brazilian Journal of Production Engineering, 8(1), 92-106.

Atualmente, encontrar esses biomarcadores preditivos (farmacogenômicos, transcricionais e proteômicos) é considerada à busca pelo Santo Graal para o tratamento do choque séptico. Todavia, esses estudos com biomarcadores enfrentam uma grande barreira que é tempo para escolha de qual tecido deve ser retirado a amostra, em virtude da velocidade com a qual o choque séptico evolui. Consequentemente, apesar do uso potencial de biomarcadores preditivos no choque séptico, a Surviving Sepsis Campaign não recomenda nenhum deles (Russell, 2018).

No que diz respeito às limitações e barreiras, a análise dos artigos pode ter sido prejudicada pela não utilização de um instrumento para análise da qualidade dos artigos, bem como a quantidade de amostras nos artigos, que variavam significativamente. Além disso, outra limitação foi a busca e a interpretação dos dados obtidos ter sido feita por um único pesquisador, apesar de que a verificação da veracidade dos fatos foi feita realizada por dois avaliadores de forma conjunta.

\section{CONCLUSÃO}

De acordo com os dados da análise dos artigos, concluiu-se que o uso dos corticosteroides na terapia adjuvante do choque séptico não apresenta evidência clara na redução, ou na melhor das hipóteses, uma pequena redução, da mortalidade. No entanto, esses medicamentos estão consideravelmente relacionados à diminuição do tempo para retirada da ventilação mecânica e do tempo em cuidados intensivos, assim como associa-se a uma reversão mais rápida do choque séptico. Com isso, essa terapêutica evidencia uma diminuição no tempo de internamento em unidade de terapia intensiva (UTI), principalmente quando trata-se de pacientes em estado crítico.

Além disso, a análise dos artigos evidenciou que apesar da grande variabilidade de efeitos adversos que os corticosteroides podem causar no organismo humano, as adversidades mais frequentes são aquelas com menor repercussão clínica para os pacientes, como por exemplo, a hiperglicemia.

Contudo, a terapia adjuvante com corticosteroides não deve ser instituída como prática rotineira para o tratamento do choque séptico, visto que os pacientes candidatos a esse tratamento são aqueles com maior gravidade e/ou que não respondem primeiramente a ressuscitação com fluidos e drogas vasoativas. Por isso, o uso dessas medicações deve ser individualizado, levando-se em consideração o fenótipo e o genótipo de cada paciente.

\section{REFERÊNCIAS}

Allen, J. M., Feild, C., Shoulders, B. R., \& Voils, S. A. (2019). Recent updates in the pharmacological management of sepsis and septic shock: a systematic review focused on fluid resuscitation, vasopressors, and corticosteroids. Annals of Pharmacotherapy, 53(4), 385-395. https://doi.org/10.1177/1060028018812940

Angurana, S. K. \& Kumar, P. (2017). Assessing feasibility of randomized controlled trials of corticosteroids in pediatric septic shock in developed countries: only half the answer to the $\begin{array}{lllll}\text { problem. Pediatric Critical } & \text { Care }\end{array}$ https://doi.org/10.1097/PCC.0000000000001310 
Citação (APA): Leite, W. F. J., Medeiros, O. B., de, Filho, \& Sousa, M. N. A., de. (2022). Eficácia do uso dos corticosteroides como terapia adjuvante no choque séptico. Brazilian Journal of Production Engineering, 8(1), 92-106.

Annane, D. (2019). Why my steroid trials in septic shock were "positive". Critical care medicine, 47(12), 1789-1793. https://doi.org/10.1097/CCM.0000000000003889

Antcliffe, D. B. \& Gordon, A. C. (2019). Why understanding sepsis endotypes is important for steroid trials in septic shock. Critical care medicine, 47(12), 1782-1784. https://doi.org/10.1097/CCM.0000000000003833

Argent, A. C. \& Kissoon, N. (2017). Randomized Clinical Trials of Corticosteroids in Septic Shock: Possibly Feasible, But Will They or Should They Change My Practice? Pediatric critical care medicine, 18(6), 589-590. https://doi.org/10.1097/PCC.0000000000001138

Bentzer, P., Fjell, C., Walley, K. R., Boyd, J., \& Russell, J. A. (2016). Plasma cytokine levels predict response to corticosteroids in septic shock. Intensive care medicine, 42(12), 19701979. https://doi.org/10.1007/s00134-016-4338-z

Briegel, J., Bein, T., \& Möhnle, P. (2017). Update on low-dose corticosteroids. Current Opinion in Anesthesiology, 30(2), 186-191. https://doi.org/10.1097/ACO.0000000000000442

Cecconi, M., Evans, L., Levy, M., \& Rhodes, A. (2018). Sepsis and septic shock. The Lancet, 392(10141), 75-87. https://doi.org/10.1016/S0140-6736(18)30696-2

Cohen, J., \& Venkatesh, B. (2019). Adjunctive corticosteroid treatment in septic shock. Anesthesiology, 131(2), 410-419. https://doi.org/10.1097/ALN.0000000000002604

Costanzo, L. S. (2018). Fisiologia. 6. ed. Rio de Janeiro: Elsevier Editora Ltda.

Cvijanovich, N. Z., Anas, N., Allen, G. L., Thomas, N. J., Bigham, M. T., Weiss, S. L., ... \& Wong, H. R. (2017). Glucocorticoid receptor polymorphisms and outcomes in pediatric septic shock. Pediatric critical care medicine: a journal of the Society of Critical Care Medicine and the World Federation of Pediatric Intensive and Critical Care Societies, 18(4), 299. https://doi.org/10.1097/PCC.0000000000001058

Darmaros, L. F., Delgado, A. F., \& Carvalho, W. B. D. (2016). Corticosteroids in septic shock: What should the decision in pediatrics be? Revista da Associação Médica Brasileira, 62, 482-484. https://doi.org/10.1590/1806-9282.62.06.482

El-Nawawy, A., Khater, D., Omar, H., \& Wali, Y. (2017). Evaluation of early corticosteroid therapy in management of pediatric septic shock in pediatric intensive care patients: a randomized clinical study. The Pediatric infectious disease journal, 36(2), 155-159. https://doi.org/10.1097/INF.0000000000001380

Fang, F., Zhang, Y., Tang, J., Lunsford, L. D., Li, T., Tang, R., ... \& You, C. (2019). Association of corticosteroid treatment with outcomes in adult patients with sepsis: a systematic review and meta-analysis. JAMA internal medicine, 179(2), 213-223. https://doi.org/10.1001/jamainternmed.2018.5849

Garza, M. I., Zapata, M. P., Bilbao, I. G., Martínez, C. F., Horno, R. A., \& Pomes, G. G. (2018). Administración de corticoides a los pacientes con sepsis grave y mejora de su mortalidad intrahospitalaria: Una revisión sistemática. Enfermería Global, 17(4), 612-639. https://doi.org/10.6018/eglobal.17.4.321571

Gibbison, B., López-López, J. A., Higgins, J. P., Miller, T., Angelini, G. D., Lightman, S. L., \& Annane, D. (2017). Corticosteroids in septic shock: a systematic review and network metaanalysis. Critical Care, 21(1), 1-8. https://doi.org/10.1186/s13054-017-1659-4

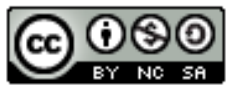


Citação (APA): Leite, W. F. J., Medeiros, O. B., de, Filho, \& Sousa, M. N. A., de. (2022). Eficácia do uso dos corticosteroides como terapia adjuvante no choque séptico. Brazilian Journal of Production Engineering, 8(1), 92-106.

Golan, D.E., Tashjian, A.H., Armstrong, E.J., \& Armstrong, A.W. (2018). Princípios de farmacologia: a base fisiopatológica da farmacoterapia. 3. ed. Rio de Janeiro: Guanabara Koogan.

Hall, J.E. \& Guyton, A.C. (2017). Guyton e Hall fundamentos da fisiologia. 13. ed. Rio de Janeiro: Elsevier Editora Ltda.

Hyvernat, H., Doyen, D., Barel, R., Kaidomar, M., Goubaux, B., Pradier, C., ... \& Bernardin, G. (2018). Is inappropriate response to cosyntropin stimulation test an indication of corticosteroid resistance in septic shock? Shock: Injury, Inflammation, and Sepsis: Laboratory and Clinical Approaches, 49(5), 543-550. https://doi.org/10.1097/SHK.0000000000001014

Instituto Latino Americano de Sepse (ILAS) (2018). Implementação de protocolo gerenciado de sepse protocolo clínico: Atendimento ao paciente adulto com sepse/choque séptico. São Paulo: $\quad$ ILAS. $14 \mathrm{p}$ Recuperado de https://www.hcrp.usp.br/revistaqualidadehc/edicaoselecionada.aspx?Edicao=6

Ismail, J. \& Jayashree, M. (2018). Advances in the Management of Pediatric Septic Shock: Old Questions, New Answers. Indian pediatrics, 55(4).

Jorge, R. L. N., Lourenço, L. A., Vieira, L. H. D. A., Santana, M. N., \& Pedroso, E. R. P. (2016). Choque séptico. Rev Med Minas Gerais, 26(4), 9-12. https://doi.org/10.5935/2238$\underline{3182.20160040}$

Law, A. C., Sahetya, S., Walkey, A. J., \& Delaney, A. (2018). Low-dose corticosteroids and septic shock resolution: implications for randomized trial design? Intensive care medicine, 44(8), 1366-1367. https://doi.org/10.1007/s00134-018-5265-y

Lee, C. T., \& Yeh, Y. C. (2018). The role of corticosteroid in septic shock patients: The authors reply. Journal of critical care, 43, 386. https://doi.org/10.1016/j.jcrc.2017.11.016

Lemieux, S. M., Levine, A. R., \& Gritsenko, D. (2019). Was Adjunctive Corticosteroid Treatment in Critically Ill Patients with Septic Shock (ADRENAL) Insufficient? Critical care medicine, 47(12), e1035-e1035. https://doi.org/10.1097/ccm.0000000000003980

Lian, X. J., Huang, D. Z., Cao, Y. S., Wei, Y. X., Lian, Z. Z., Qin, T. H., ... \& Wang, S. H. (2019). Reevaluating the role of corticosteroids in septic shock: an updated meta-analysis of randomized controlled trials. BioMed research international. https://doi.org/10.1155/2019/3175047

Liu, A., \& Menon, K. (2018). Contributions of a survey and retrospective cohort study to the planning of a randomised controlled trial of corticosteroids in the treatment of paediatric septic shock. Trials, 19(1), 1-8. https://doi.org/10.1186/s13063-018-2664-X

Long, B., \& April, M. D. (2018). Do corticosteroids improve mortality or shock reversal in patients with septic shock? Annals of Emergency Medicine, 71(1), 34-36. https://doi.org/10.1016/j.annemergmed.2017.05.025

Long, B., \& Koyfman, A. (2017). Controversies in corticosteroid use for sepsis. The Journal of emergency medicine, 53(5), 653-661. https://doi.org/10.1016/j.jemermed.2017.05.024

Mendes, K. D. S., Silveira, R. C. D. C. P., \& Galvão, C. M. (2008). Revisão integrativa: método de pesquisa para a incorporação de evidências na saúde e na enfermagem. Texto \& contexto-enfermagem, 17, 758-764. https://doi.org/10.1590/S0104-07072008000400018

Menon, K., \& McNally, J. D. (2018). Corticosteroids in pediatric septic shock are helpful. Critical care medicine, 46(4), 635-636. https://doi.org/10.1097/CCM.0000000000002979 
Citação (APA): Leite, W. F. J., Medeiros, O. B., de, Filho, \& Sousa, M. N. A., de. (2022). Eficácia do uso dos corticosteroides como terapia adjuvante no choque séptico. Brazilian Journal of Production Engineering, 8(1), 92-106.

Meyer, E. J., Nenke, M. A., Rankin, W., Lewis, J. G., Konings, E., Slager, M., ... \& Torpy, D. J. (2019). Total and high-affinity corticosteroid-binding globulin depletion in septic shock is associated with mortality. Clinical endocrinology, 90(1), 232-240. https://doi.org/10.1111/cen.13844

Moskowitz, A., Yankama, T., Andersen, L. W., Huang, D. T., Donnino, M. W., \& Grossestreuer, A. V. (2019). Ascorbic Acid, Corticosteroids and Thiamine in Sepsis (ACTS) protocol and statistical analysis plan: a prospective, multicentre, double-blind, randomised, placebo-controlled clinical trial. BMJ open, 9(12), e034406. https://doi.org/10.1136/bmjopen2019-034406

O’Hearn, K., McNally, D., Choong, K., Acharya, A., Wong, H. R., Lawson, M., ... \& Menon, K. (2016). Steroids in fluid and/or vasoactive infusion dependent pediatric shock: study protocol for a randomized controlled trial. Trials, 17(1), 1-10. https://doi.org/10.1186/s13063$\underline{016-1365-6}$

Pourmand, A., Whiteside, T., Yamane, D., Rashed, A., \& Mazer-Amirshahi, M. (2019). The controversial role of corticosteroids in septic shock. The American journal of emergency medicine, 37(7), 1353-1361. https://doi.org/10.1016/j.ajem.2019.04.045

Ramamoorthy, S., \& Cidlowski, J. A. (2016). Corticosteroids: mechanisms of action in health and disease. Rheumatic Disease Clinics, 42(1), 15-31. https://doi.org/10.1016/j.rdc.2015.08.002

Ramanan, M., Cohen, J., \& Venkatesh, B. (2019). Steroids and Sepsis: the Debate Continues. International anesthesiology clinics, $\quad$ 57(2), 17-30. https://doi.org/10.1097/AIA.0000000000000220

Rhodes, A., Evans, L. E., Alhazzani, W., Levy, M. M., Antonelli, M., Ferrer, R., ... \& Dellinger, R. P. (2017). Surviving sepsis campaign: international guidelines for management of sepsis and septic shock: 2016. Intensive care medicine, 43(3), 304-377. https://doi.org/10.1007/s00134-017-4683-6

Ritter, J.M., Flower, R., Henderson, G., Loke, Y.k., MacEwan, D., \& Rang, H.P. (2020) Rang \& Dale: farmacologia. 9. ed. Rio de Janeiro: Guanabara Koogan.

Russell, J. A. (2018). When and how to use predictive biomarkers for corticosteroid treatment of septic shock. Critical Care, 22(1), 1-3. https://doi.org/10.1186/s13054-018-2254-Z

Russell, J. A., \& Sevransky, J. (2019). Toward increased understanding of the steroid controversy in septic shock. Critical care medicine, 47(12), 1677-1679. https://doi.org/10.1097/CCM.0000000000004038

Salluh, J. I., \& Póvoa, P. (2017). Corticosteroids in severe sepsis and septic shock: a concise review. Shock, 47(1S), 47-51. https://doi.org/10.1097/SHK.0000000000000704

Seymour, C. W., \& Rosengart, M. R. (2015). Septic shock: advances in diagnosis and treatment. Jama, 314(7), 708-717. https://doi.org/10.1001/jama.2015.7885

Souza, M. T. D., Silva, M. D. D., \& Carvalho, R. D. (2010). Revisão integrativa: o que é e como fazer. Einstein (São Paulo), 8, 102-106. https://doi.org/10.1590/S1679$\underline{45082010 R W 1134}$

Venkatesh, B., \& Cohen, J. (2019). Why the adjunctive corticosteroid treatment in critically Ill patients with septic shock (ADRENAL) trial did not show a difference in mortality. Critical care medicine, 47(12), 1785-1788. https://doi.org/10.1097/CCM.0000000000003834 
Wen, Y., Zhu, Y., Jiang, Q., Guo, N., Cai, Y., \& Shen, X. (2019). The effectiveness and safety of corticosteroids therapy in adult critical ill patients with septic shock: a meta-analysis of randomized controlled trials. Shock, 52(2), 198-207. https://doi.org/10.1097/SHK.0000000000001202

Wong, H. R., Atkinson, S. J., Cvijanovich, N. Z., Anas, N., Allen, G. L., Thomas, N. J., ... \& Lindsell, C. J. (2016). Combining prognostic and predictive enrichment strategies to identify children with septic shock responsive to corticosteroids. Critical care medicine, 44(10), e1000. https://doi.org/10.1097/CCM.0000000000001833

Yang, J., \& Sun, S. (2020). Controversies in the application of corticosteroids for pediatric septic shock treatment: a preferred reporting items for systematic reviews and meta-analysis$\begin{array}{lll}\text { compliant updated meta-analysis. } & \text { Medicine, }\end{array}$ https://doi.org/10.1097/MD.0000000000020762

Zhou, X., Ye, Y., Li, J., \& Tang, G. (2018). The role of corticosteroid in septic shock patients. Journal of critical care, 43, 384-385. https://doi.org/10.1016/j.jcrc.2017.11.015

Zimmerman, J. J. (2016). Adjunctive Corticosteroids for Contemporary Pediatric Shock: Ongoing Analysis of the Risk-Benefit Ratio. The Journal of pediatrics, 177, 911. https://doi.org/10.1016/j.jpeds.2016.06.039

Zimmerman, J. J. (2018). Corticosteroids in pediatric septic shock are not helpful. Critical care medicine, 46(4), 637. https://doi.org/10.1097/CCM.0000000000002980 